- This document has been reproduced from the best copy furnished by the organizational source. It is being released in the interest of making available as much information as possible.

- This document may contain data, which exceeds the sheet parameters. It was furnished in this condition by the organizational source and is the best copy available.

- This document may contain tone-on-tone or color graphs, charts and/or pictures, which have been reproduced in black and white.

- This document is paginated as submitted by the original source.

- Portions of this document are not fully legible due to the historical nature of some of the material. However, it is the best reproduction available from the original submission.

Produced by the NASA Center for Aerospace Information (CASI) 
$X-953-77-154$

$$
\operatorname{Tm} x-71371
$$

PREPRINT

\title{
SIMULATION OF RADIATION FROM LIGHTNING RETURN STROKES: THE EFFECTS OF TORTUOSITY
}

(NASA-TM-X-71371) SIMULATION OF RADIATION

FROM LIGHTNING RETURN STROKES: THE EFFECTS

OF TOBTUOSITY (NASA) $32 \mathrm{P}$ HC A03/MF A01

$\begin{array}{lll}\text { CSCL 04A } & & \text { Uncilas } \\ & \text { G3/46 } & 46014\end{array}$

\author{
D. M. LEVINE \\ R. MENEGHINI
}




\section{SIMULATION OF RADIATION FROM \\ LIGHTNING RETURN STROKES: \\ THE EFFECTS OF TORTUOSITY}

D. M. LeVine

R. Meneghini

GODDARD SPACE FLIGHT CENTER

Greenbelt, Maryland 


\title{
SIMULATION OF RADIATION FROM
}

\section{LIGHTNING RETURN STROKES:}

\section{THE EFFECTS OF TORTUOSITY}

\begin{abstract}
A Monte Carlo simulation has been developed for the electromagnetic fields radiated from a tortuous lightning channel. This was done using a piecewise linear model for the chamel and employing for each element the field radiated by a traveling wave on an arbitrarily oriented filament over a conducting plane. The simulation reproduces experimental data reasonably well and has been used to study the effects of tortuousity on the fields radiated by return strokes.

Tortuosity can significantly modify the radiated waveform, tending to render it less representative of the current pulse and more nearly unipolar than one would expect based on the theory for a long straight channel. In the frequency domain the effect of tortuosity is an increase in high frequency energy as compared with an equivalent straight channel. The extent of this increase depends on the mean length of the elements comprising the channel and can be significant.
\end{abstract}




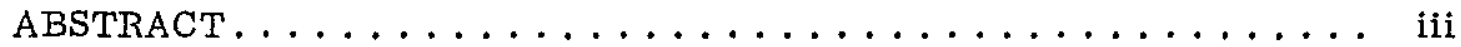

Introduction. . . . . . . . . . . . . . . . . . . 1

Comparison with Measured Data. ............... 9

Effects of Tortuosity .................... 11

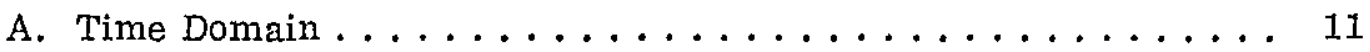

B. Frequency Domain. ..................... 13

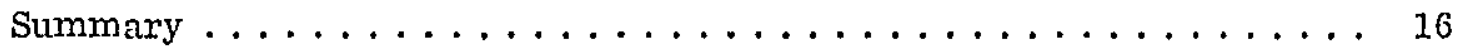

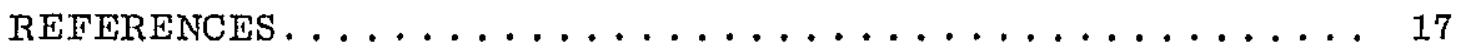




\section{SIMULATION OF RADIATION FROM}

LIGHTNING RETURN STROKES:

THE EFFECTS OF TORTUOSITY

Introduction

A Monte Carlo simulation has been developer for the electromagnetic fields radiated from a tortuous lightning channel by using a piecewise linear model for the channel and assuming a traveling wave ("transmission line" model) for the current in each element. This simulation has been developed to study the effects of tortuosity on the electromagnetic radiation emitted from return strokes in cloud-to-ground lightning, and is also proving useful in evaluating the effect of other lightning parameters, such as current pulse shape and propagation velocity, on the radiated waveforms.

The simulation is performed by numerically creating a lightning channel out of linear segments whose length and orientation are chosen in accordance with a specified statistical model. It is assumed that a current pulse with shape $I_{0}(t)$ propagates along each segment at velocity, $v$ : that is $I(t)=I_{0}(t-\hat{l} \cdot \bar{r} / v)$. Typically this pulse begins at the base of the channel and propagates up toward the cloud, possibly dividing and changing velocity at branches. The solution for the radiated fields is obtained by summing the fields radiated from each of the linear elements, keeping proper track of the phase of the current driving each element 
as the current pulse moves along the channel. It is assumed that the ground plane above which the chamel is situated is perfectly conducting $(\sigma=\infty)$ so that for each element there is also an image element below the plane. The basic geometry is illustrated in Figure 1.

The analytic problem central to the simulation is to determine the electromagnetic fields radiated by the arbitrarily oriented linear current filaments from which the channel is constructed. The solution is required in the case of a traveling (propagating) current pulse, making the problem analogous to calculating radiation from an arbitrarily oriented traveling wave antenna positioned over a conducting plane. Two forms of this solution have been obtained.

The first solution, and the one used to produce most of the results which will be presented here, employs the fraunhofer (i.e. far field) approximation to obtain closed form integrals for the fields. This solution is obtained for arbitrary $I_{0}(t)$ and arbitrary filament orientation subject only to the constraint that the current propagates along the channel. The velocity of propagation as well as location of the observer are also arbitrary. The time domain form of this solution in the special case of an observer located on the conducting surface is given below in Equations 1 . The general form for the solution and details of its derivation are presented in LeVine and Meneghini (1976a).

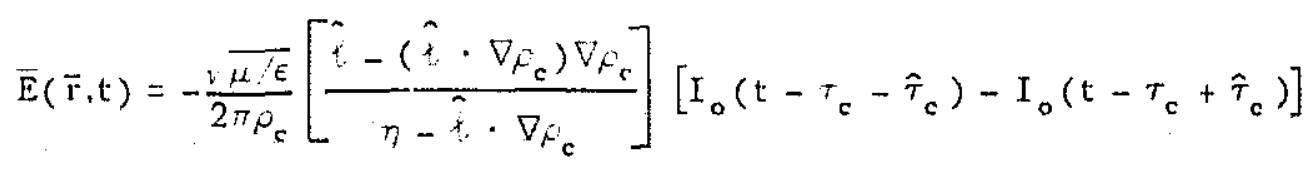




$$
\overline{\mathrm{H}}(\bar{r}, \mathrm{t})=\frac{1}{2 \pi \rho_{\mathrm{c}}}\left[\frac{\nabla \rho_{\mathrm{c}} \times \hat{l}}{\eta-\hat{l} \cdot \nabla \rho_{\mathrm{c}}}\right]\left[\mathrm{I}_{\mathrm{o}}\left(\mathrm{t}-\tau_{\mathrm{c}}-\hat{\tau}_{\mathrm{c}}\right)-\mathrm{I}_{\mathrm{o}}\left(\mathrm{t}-\tau_{\mathrm{c}}+\hat{\tau}_{\mathrm{c}}\right)\right]
$$

where $\tau_{c}=\rho_{c} / \mathrm{c}-\hat{l} \cdot \bar{r}_{\mathrm{c}} / \mathrm{V}$ and $\hat{\tau}_{\mathrm{c}}=L_{\mathrm{L}} / 2 \mathrm{~V}-(\mathrm{L} / 2 \mathrm{c})\left(\hat{l} \cdot \nabla \rho_{\mathrm{c}}\right)$; where $\rho_{\mathrm{c}}$ is the distance from filament center to the observer and $I$ is the length of the filament; where the unit vector $\hat{l}$ points in the direction of propagation along the filament and $\bar{x}_{c}$ is the position vector of the center of the filament; and where $\eta=\mathrm{c} / \mathrm{v}$ is an index of refraction. The first term in $\hat{\tau}_{\mathrm{c}}$ is just the time required for the current waveform to propagate halt the length of the filament and the second term is the binomial correction to the distance from observer to filament center needed to account for the slight differences in location of the end points of the filament relative to its center. (See insert in Figure 1.)

The second solution obtained for the electromagnetic problem has the unique attribute of being exact: That is, no mathematical approximateions are necessary in arriving at closed form solutions to Maxwell's equations. However, the solution applies only to the case where the velocity of propagation, $v$, of the current pulse is equal to $c$, the velocity of propagation of light in the ambient medium. This solution is an extension, to include arbitrary filament orientation and the effects of the ground plane, of a solution presented in the time domain by Schelkunoff (Schelkunoff, 1952) and earlier in its frequency domain form by Pistolkors (1929) and Beckmann (1931). The form this solution takes in the time domain for an observer located on the surface is: 


$$
\begin{aligned}
E(\bar{r}, \mathrm{t})= & \frac{\sqrt{\mu / \epsilon}}{2 \pi}\left\{\frac{\bar{\epsilon}\left(\rho_{\mathrm{b}}\right)}{\rho_{\mathrm{b}}} \mathrm{I}_{\mathrm{o}}\left(\mathrm{t}-\tau_{\mathrm{b}}\right)-\frac{\bar{\epsilon}\left(\rho_{\mathrm{a}}\right)}{\rho_{\mathrm{a}}} \mathrm{I}_{\mathrm{o}}\left(\mathrm{t}-\tau_{\mathrm{a}}\right)\right\} \\
& -\frac{1}{2 \pi \epsilon}\left\{\frac{\nabla_{\mathrm{b}}}{\rho_{\mathrm{b}}^{2}} \int_{0}^{\mathrm{t}} \mathrm{I}_{\mathrm{o}}\left(\xi-\tau_{\mathrm{b}}\right) \mathrm{d} \xi-\frac{\nabla_{\mathrm{a}}}{\rho_{\mathrm{a}}^{2}} \int_{0}^{\mathrm{t}} \mathrm{I}_{\mathrm{o}}\left(\xi-\tau_{\mathrm{a}}\right) \mathrm{d} \xi\right\} \\
\overrightarrow{\mathrm{H}}(\overline{\mathrm{r}}, \mathrm{t})= & \frac{\bar{h}\left(\rho_{\mathrm{b}}\right)}{2 \pi \rho_{\mathrm{b}}} \mathrm{I}_{\mathrm{o}}\left(\mathrm{t}-\tau_{\mathrm{b}}\right)-\frac{\overline{\mathrm{h}}\left(\rho_{\mathrm{a}}\right)}{2 \pi \rho_{\mathrm{a}}} \mathrm{I}_{\mathrm{o}}\left(\mathrm{t}-\tau_{\mathrm{a}}\right)
\end{aligned}
$$

where for $i=a, b$ :

$$
\begin{aligned}
& \bar{\epsilon}\left(\mu_{\mathrm{i}}\right)=\sqrt{\frac{1+\hat{\ell} \cdot \nabla \rho_{\mathrm{i}}}{1-\hat{\ell} \cdot \nabla_{\rho_{\mathrm{i}}}}} \hat{\epsilon}\left(\rho_{\mathrm{i}}\right) \\
& \hat{\varepsilon}\left(p_{\mathrm{i}}\right)=\frac{\hat{l}-\left(\hat{l} \cdot \nabla p_{\mathrm{i}}\right) \nabla p_{\mathrm{i}}}{\sqrt{1-\left(\hat{l} \cdot \nabla p_{\mathrm{i}}\right)^{2}}} \\
& \bar{h}\left(\rho_{\mathrm{i}}\right)=\sqrt{\frac{1+\hat{l} \cdot \nabla_{\rho_{\mathrm{i}}}}{1-\hat{l} \cdot \nabla_{\mu_{i}}}} \hat{h}\left(\mu_{\mathrm{i}}\right) \\
& \hat{h}\left(\rho_{\mathrm{i}}\right)=\frac{\nabla \rho_{\mathrm{i}} \times \hat{l}}{\sqrt{1-\left(\hat{l} \cdot \nabla \rho_{\mathrm{i}}\right)^{2}}} \\
& \tau_{i}=\left(\hat{\rho}_{i}-\hat{X} \cdot \bar{r}_{i}\right) / c
\end{aligned}
$$

and where $f_{\mathrm{a}, \mathrm{b}}$ are the distances from the ends of the filament to the observer (Figure 1) and $\overline{\mathrm{r}}_{\mathrm{n}, \mathrm{b}}$ are the position vectors which locate the ends of the filament with respect to the reference coordinate system. The general form of the solution and details of its derivation are to be found in LeVine and Meneghini $(1976 \mathrm{a}, \mathrm{b})$ This exact solution has been included as part of the moclel both to keep track of the effect of the approximations made in obtaining the fraunhofer form, and also 
to give some insight into the near field behaviour of the radiated fields, the region where the fraurihofer approximation is inappropriate.

The current waveform assumed in this simulation is a compound exponential model based on the form proposed by Uman (1969), but modified slightly to be continuous at $t=0$. The model used here is:

$$
I_{0}=I_{0}\left[e^{-a t}-e^{-\beta t}\right]+I_{1}\left[e^{-\gamma t}-e^{-\delta t}\right]
$$

where, for a typical lightning return stroke the parameters are [Uman, 1969]:

$$
\begin{aligned}
a & =2.0 \times 10^{4} \\
\beta & =2.0 \times 10^{5} \\
y & =1.0 \times 10^{3} \\
\delta & =2.0 \times 10^{4} \\
I_{0} & =30 \mathrm{ka} \\
I_{1} & =2.5 \mathrm{ka}
\end{aligned}
$$

The first two exponentials in Equation 4 represent the main current pulse in a form proposed by Bruce and Golde (1941) with parameters suggested by Dennis and Pierce (1964). The third term, $I_{1} \exp (-\gamma / t)$, represents intermediate current (Uman, 1969); and the remaining exponential, $I_{1} \exp (-\delta t)$, has been added to achieve continuity at $t=0$ and doesn't, otherwise, significantly alter the current pulse. (See Figure 2 for the shape of the current pulse.) The simulation operates independently of the actual current waveform, any form being aliowed as long as it propagates along the channel; however, most of the clata obtained to date has been based on the preceeding waveform since it appears to reasonably represent a composite of reported measurements (Uman, 1969; Dennis and Pierce, 1964). 
An example of typical data produced by the simulation is shown in Figures 3 and 4. Figure 3 shows the three dimensional channel generated by the computer including, in this case, branches. To generate the channel, the number and statistics of the main channel elements and branches are specified and then the machine produces the particular realization by choosing numbers for the length and orientation of the elements in accordance with the assigned statistics. The number of branches is assigned, but their location is chosen stochastically by the computer. The projection of the channel on orthogonal planes is shown in the two figures.

Once the channel has been generated, the computer calculates both the temporal history of the electromagnetic fields produced by a current pulse propagating along this channel and also the Fourier transform (spectrum) of the radiated waveform. This is done using the solutions given in Equations 1 or 2 and their frequency domain equivalent, and employing an assigned current waveform such as given by Equation 4. The major computational problem is keeping track of the proper phase of the current in each of the many elements which may be radiating at a given instant.

An example of the temporal history and spectrum of the electric field waveform radiated from the chamel shown in Figure 3 and are given in Figure 4 . The current waveform is as given in Equation 4 with $\mathrm{v}=\mathrm{c}$, and the observer is located on the $y$-axis $100 \mathrm{~km}$ from the origin. Just as the chamnel appears clifferent to observers located at different points so the radiated waveform measured by 
observers at different locations is different. However, the shape illustrated in Figure 4 is typical of the waveform seen by an observer located on the surface. The bottom illustration in Figure 4 represents the spectrum (magnitude of the Fourier transform) of the radiated electric field shown at the top. 'The shape of the spectrum is typical of an essentially vertical chanrel with the observer on the surface; However, the spectrum depends strongly on such parameters as channel length and orientation, velocity of propagation, and shape of the current pulse. (See LeVine and Meneghini, 1967a for examples and also Figure 9). The high frequency asymptote in this example is representative of data obtained with a current waveform as described in Equation 4. But at high frequency this data is under sampled (because of size limitations in the graph) so that the oscillating character evident at high frequency is not quantitatively correct. The number and depth of the nulls are not faithfully represented, although the asymptote of the peak value is correctly indicated. The effect of aliasing is especially evident in the spectra shown in Figure 5 . On the other hand, the first few "oscillations" (near $100 \mathrm{kHz}$ ) are properly represented.

The radiated waveform and spectrum presented in Figure 4 have been produced using the fraunhofer solution. The exact solution produces results which are ostensibly the same in the time domain because the observer is in the far field. However, in the frequency domain there is an essential difference at very low frequencies. This is because the fraunhofer solution fails for an observer closer than several wavelengths from the filament. The fraunhofer solution 
predicts a decrease in spectrum proportional to frequency in the low frequency limit whereas the proper behaviour begins to increase as (frequency) ${ }^{-1}$ at low frequency. Thus in Figure 4, the proper spectrum would show an increase at frequencies less than about $1 \mathrm{kHz}$. For frequency above about $1 \mathrm{kHz}$ both exact and fraunhofer solution agree very well. This difference is illustrated in Figure 5 where the spectrum for both the exact and fraunhofer solutions are plotted for an observer on the surface and $100 \mathrm{~km}$ away from a vertical straight element. The current waveform is as given in Equation 4 and the velocity of propagation is $\mathrm{v}=\mathrm{c}$ (necessary for comparison). 
Comparison with Measured Data:

The electric field waveform predicted by the simulation will depend on both the particular realization of the channel which happens to be generated, as well as on the various parameters with which the simulation is initialized (i.e. the velocity of propagation, current waveform, scale of tortuosity, number and structure of branches and location of the observer). In fact, one of the potential uses of the simulation is in studying how these various parameters affect the radiated waveform. By so doing one, perhaps, can learn to extract channel physics from the radiated waveform. On the other hand, a comparison with data can also be used as a check on the model, because one would expect that with reasonable choices of initial parameters the simulation should produce electric field waveforms which are representative of measured data.

A comparison of selected simulated and measured electric field waveforms is shown in Figures 6 and 7. The measured data (lower figures) are electric field waveforms radiated from first return strokes during cloud-to-ground lightning flashes observed at Kennedy Space Center, Florida. These data were obtained during the Thunderstorm Research Project (Pierce, 1976) at KSC during July 1976 using an electric field measuring system (fast field change meter) provided by the University of Arizona (Krider, et al., 1976). The data were stored digitally and then reconstructed on an oscilloscope so that plotographs could be made. The data presented here are representative of first return strokes observed at KSC. Examples of the simulation are shown at the top of 
each Figure. The simulated waveform in Figure 6 was produced from the channel shown in Figure 3 with the observer at $100 \mathrm{~km}$ along the y-axis. The simulated waveform shown in Figure 7 was produced from a channel similar to the one shown on the bottom of Figure 8. There were no branches in this channel and the observer was $100 \mathrm{~km}$ away from the channel on the $\mathrm{x}$-axis. However, in this latter case, the current waveform was made more peaked by letting $a=6 \times 10^{4}$ and $\beta=10^{5}$; also the velocity of propagation of the current pulse was made to change at about $1.2 \mathrm{~km}$ such that the velocity was $\mathrm{v}=\mathrm{c}$ for the first $1.2 \mathrm{~km}$ and $\mathrm{v}=\mathrm{c} / 2$ thereafter. The increased structure in the radiated waveform in Figure 7 as compared to that in Figure 6 is primarily the result of the modified current pulse shape.

The comparison in Figures 6 and 7 are two representative cases. The comparison is not meant to be statistically significant, which would require large samples of unbiased clata, but only to show that the simulation can produce qualitiztively correct data. Figures 6 and 7 show that assuming reasonable initial parameiers the general features of the simulated waveform are in keeping with observed data. Obviously, many other checks have been made for consistency and correctness of the simulation. 


\section{Effects of Tortuousity}

The effect of tortuosity on the radiated waveform is especially appropriate for study with the simulation because all parameters can be fixed and only the channel geometry allowed to vary. This has been done here by creating a statistical sample of channels under fixed initial parameters and examining the radiation from each. The results are discussed below first as they appear in the time domain and then in the frequency domain.

\section{A. Time Domain}

The effect of increasing tortuousity on the temporal shape of the electric field radiated from a lightning channel is illusidated in Figure 8. The figures on the left represent the electric field radiated from the channels shown on the right. In each case the current waveform is as given in Equation 4 and the pulse propagates along the channel at velocity $\mathrm{v}=\mathrm{c} / 2$. The observer is located on the $\mathrm{x}$-axis $100 \mathrm{~km}$ from the channel.

The top row of figures are the results due to a long straight vertical channel (i.e. no tortuosity). For such a channel the radiated fieids mathematically separate into two terms (see Equations 1), one of which corresponds to radiation emanating from the base of the channe-- and beginning when the pulse starts to propagate up the channel, and the second corresponding to radiation from the top of the channel but not beginning until the pulse has reached the top. The 
wave form radiated from each end point is proportional to the shape of the current pulse. Thus, initially, before the pulse reaches the top of the channel, the shape of the radiated waveform is the same as the current ptlse because only one term is contributing. The time (approximately $40 \mu \mathrm{s}$ ) at which the current pulse reaches the top of the chamel is quite evident in the upper figure. At this time the second radiation pulse begins (with identical shape but opposite sign); and the subsequent electric field waveform is their sum.

When the chamel is tortuous (lower two figures), each linear element behaves as described above. The total field at the observer is then the sum of all elements radiating at a given time. Mathematically this is equivalent to many "point" radiators located at the channel kinks (i.e. points at which the linear segments join). When the adjoining segments are nearly parallel, the radiation from the top of one nearly cancels that from the bottom of the other, and the net effect is small. This is seen in the "low" tortuousity channel shown on the middle row of graphs in Figure 8 . However, when the angle between adjoining segments is large as can happen in highly tortuous channels, the radiation from each junction can be significant. The net effect, shown for the highly tortuous channel at the bottom of Figure 8 , can alter the basic shape of the radiated waveform as inferred from the long straight chamnel. The radiated waveform loses its similarity to the current pulse and the pronounced "turning off" at the top of the channel which is apparent if the top figure becomes less significant. The process of extracting information about the shape of the current pulse from the 
the radiated waveform will become more and more difficult as tortuousity increases. Even the leading edge can be altered, rendering difficult the association of rise time as measured from the radiated waveform with that of the current pulse. They are the same until the first "kink" is reached, but not so thereafter.

\section{B. Frequency Domain}

The effects of tortuosity as discussed above in terms of the temporal shape of the radiated waveform, are also manifested in the frequency domain. Two factors dominate the effect in the frequency domain: filament orientation and filament length. The tortuous channel, as compared to the vertical straight channel, acquires an increasingly greater proportion of horizontal orientation as the tortuousity is increased due to the random orientation of the component elements. Also the more variability per unit length in a given channel, the shorter the elements must be to accommodate the frequent changes in direction.

Orientation of the channel can be an important factor determining the shape of the spectrum of the radiated electromagnetic fields. This is illustrated in Figure 9 where the spectrum of the electric field radiated from a straight (i.e. single element) vertical channel and straight horizontal channel are shown. In each case the current waveform is as given in Equations 4 with $\mathrm{v}=\mathrm{c}$, a channel length of $6 \mathrm{~km}$ (the horizontal channel is $4 \mathrm{~km}$ above the surface) and the $\mathrm{ob}-$ server $100 \mathrm{~km}$ away. The horizontal channel has relatively more energy at high frequency and less at low frequencies as compared with an otherwise identical 
vertical channel. Since the tortuous channel can be thought of as adding horizontal components to what otherwise would be a long straight vertical channel, one would expect to see a shift of energy out of the lower frequencies and toward higher frequency in the tortuous channel. It should be noted however, that this orientational effect is velocity dependent and is much less pronounced at veloom ities less than about $v=c / 2$ than shown in Figure 9.

An increase in high frequency energy is also caused by shortening the length of a given element. The spectrum scales roughly as frequency multiplied by element length (LeVine and Meneghini, 1976a) so that for a given orientation the spectrum shifts toward high frequency as the element length is decreased. In a tortuous channel one would expect both smaller mean element length and an in- . crease in relative horizontal orientation. The net effect due to both is a shift of energy toward higher frequencies. At velocities of propagation much less than $\mathrm{v}=\mathrm{c}$, element length will be the clominant effect.

This increase in higl frequency energy is illustrated in Figure 10 where the spectrum of the electric field radiated from a vertical straight channel is compared with that from an otherwise identical tortuous channel. At frequencies greater than a few hundred $\mathrm{kHz}$ the spectrum associated with the tortuous channel lies above that due to the straight vertical chamel. This shift of energy toward high frequencies can be significant, an order of magnitude or two easily being produced with reasonable lightning parameters. 
Of course, what has just been described in the frequency domain is only anothor way of looking at the behaviour of the radiated fields in the time domain. Thus, in the time domain the effect of tortuousity is an increased fluctuation of the radiated signal (per unit time). This is due to the variability in the channel oxlentation and decrease in the length of the elements. Signals with a high degree of variablity require more high frequency content for their reproduction than do more smoothly varying signals. 


\section{Summary}

A Monte Carlo simulation has been developed to model radiation from lightning return strokes. The simulation employs a piecewise linear model for the channel and assumes a current pulse which propagates along the channel. The results of the simulation compare favorably with measured data.

The simulation has been used to study the effects of tortuosity on the electric field waveforms radiated from return strokes. Tortuousity tends to make the radiated fields less representative of the current pulse and more nearly unipolar than one would expect from calculations using a straight channel (i.e. single element). The effect of tortuousity in the frequency clomain is an increase in high frequency energy and reflects the increased structure (fluctuations) in the time domain. The magnitude of this increase depends on the mean length of the elements comprising the channel. 


\section{REFERENCES}

Beckmann, R. (1931), "Calculation of Electric and Magnetic Field Strengths of Any Oscillating Straight Conductors," Proc. IRE, Vol. 19 (3), pp. 461-466.

Bruce, C. E. R. and R. H. Golde (1941), "The Lightning Discharge," JIEE (London) Vol. 88 (II), pp. 487, 505.

Demis, A. S. and E. T. Pierce (1964), "The Return Stroke of the Lightning Flash to Earth or a Source of VLF Atmospheric Radio Science, Vol. 68D(7), pp. $777-794$.

Krider, E. P., C. D. Weidman and R. C. Noggle (1976), "The Electric Fields Produced by Lightning Stepped Leaders" J. Geophys. Res., Vol. 82 (6), pp. 951-960.

LeVine, D. M. and R. Meneghini (1976a), "Radiation from a Current Filament Driven by a Traveling Wave," NASA TN-D-8302.

LeVine, D. M. and R. Meneghini (1976b), "Rxact Expressions for Electromagnetic Fields Radiated from a Lightning Return Stroke," Submitted to J. Geophys. Res. (also NASA X-953-76-272).

Pierce, E. T. (1976), "The Thunderstorm Research International Project (TRIP)$76, "$ Bull. A. M. S., Vol, 57 (10), pp. 1214-1216.

Pistolkors, A. A. (1929), "The Radiation Resistance of Beam Antennas," Proc. IRE, Vol. 17 (3), pp. 562-579. 
Schelkunoff, S. A. (1952), Advanced Antenna Theory, Jolnn Wiley and Sons, Inc., New York.

Uman, M. A. (1969), Lightning, McGraw-Hill. 


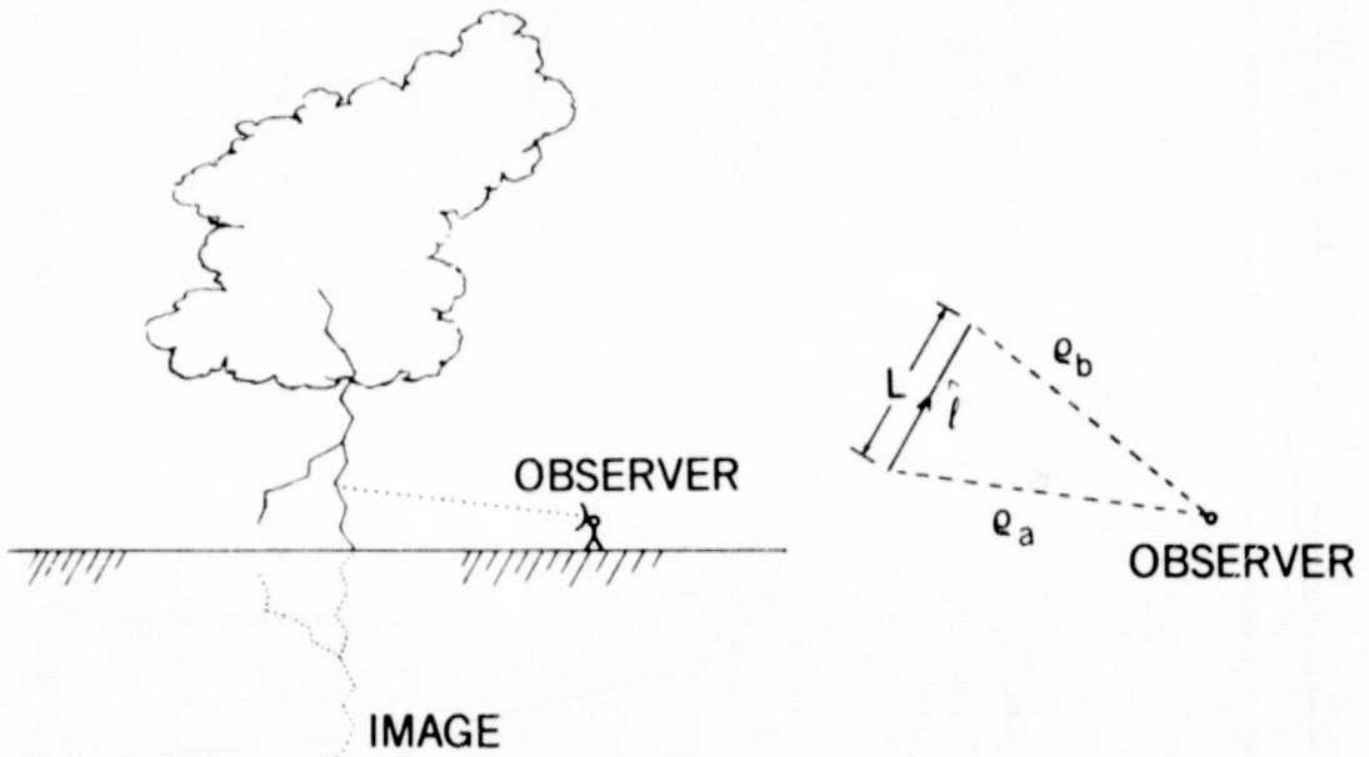

Figure 1. Simulation geometry. The insert defines variables used in writing the electromatnetic fields radiated from each channel element. 


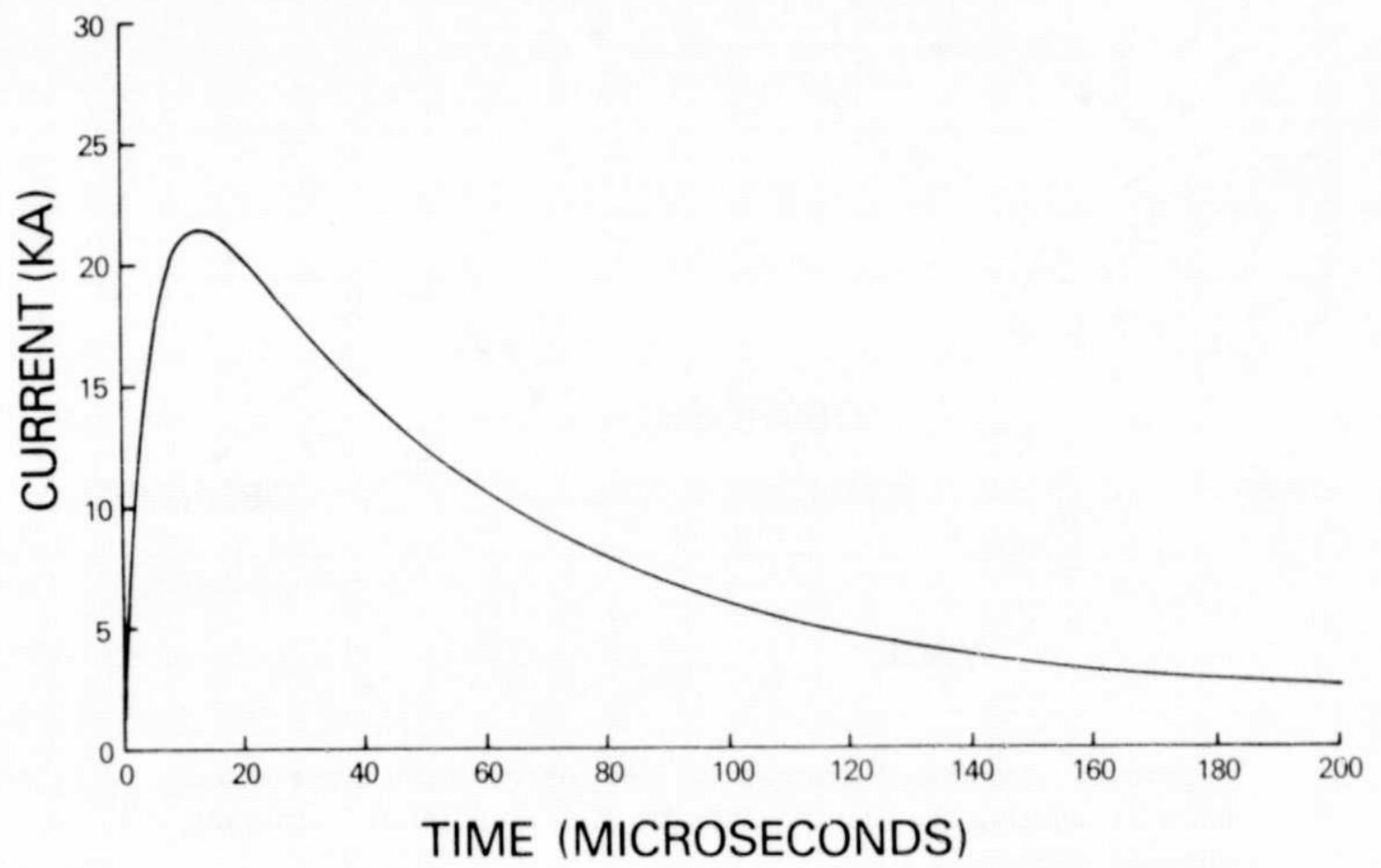

Figure 2. The current waveform used in this analysis.

The analytic form is given in Equation 4. 


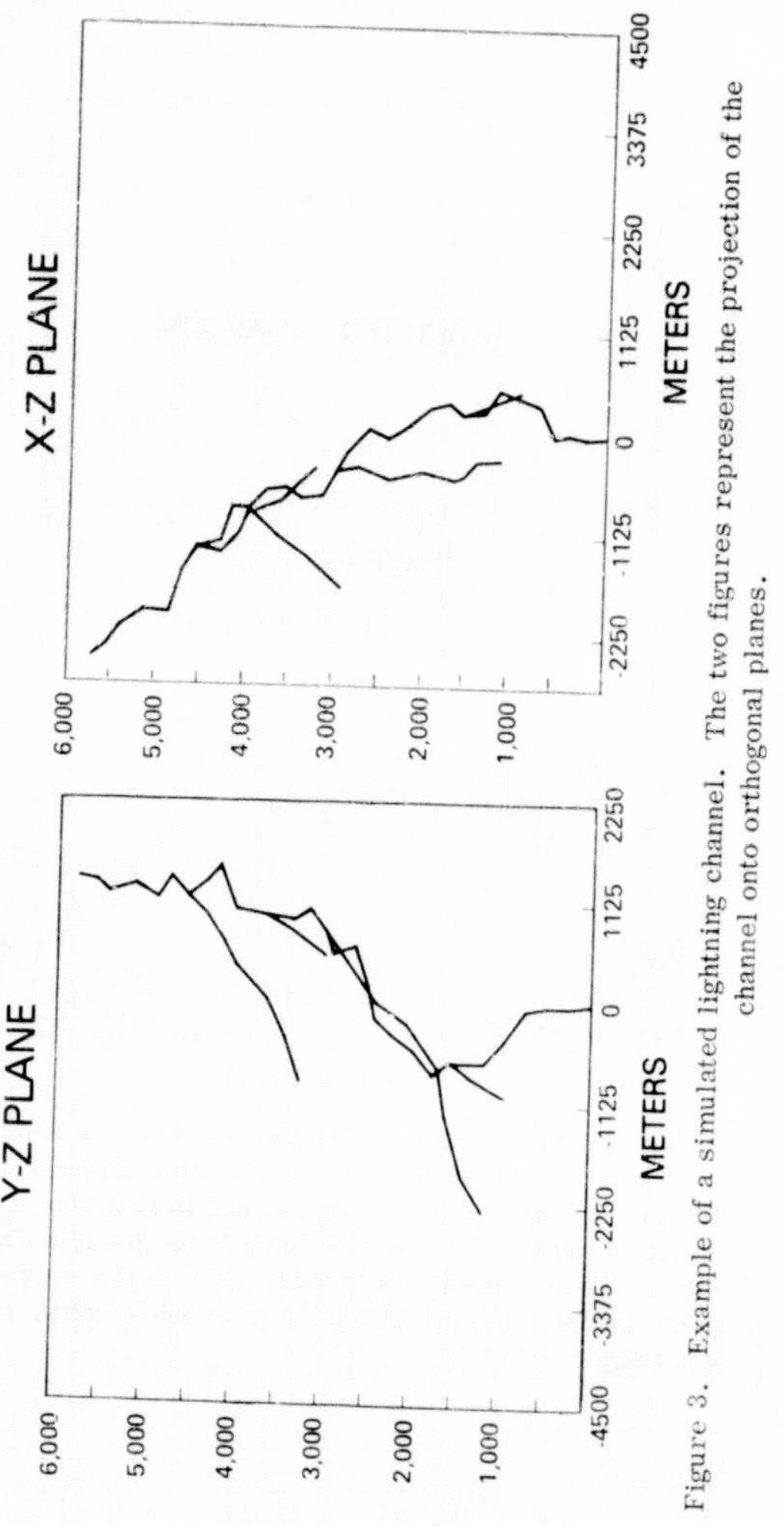

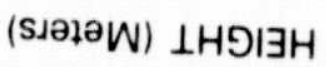



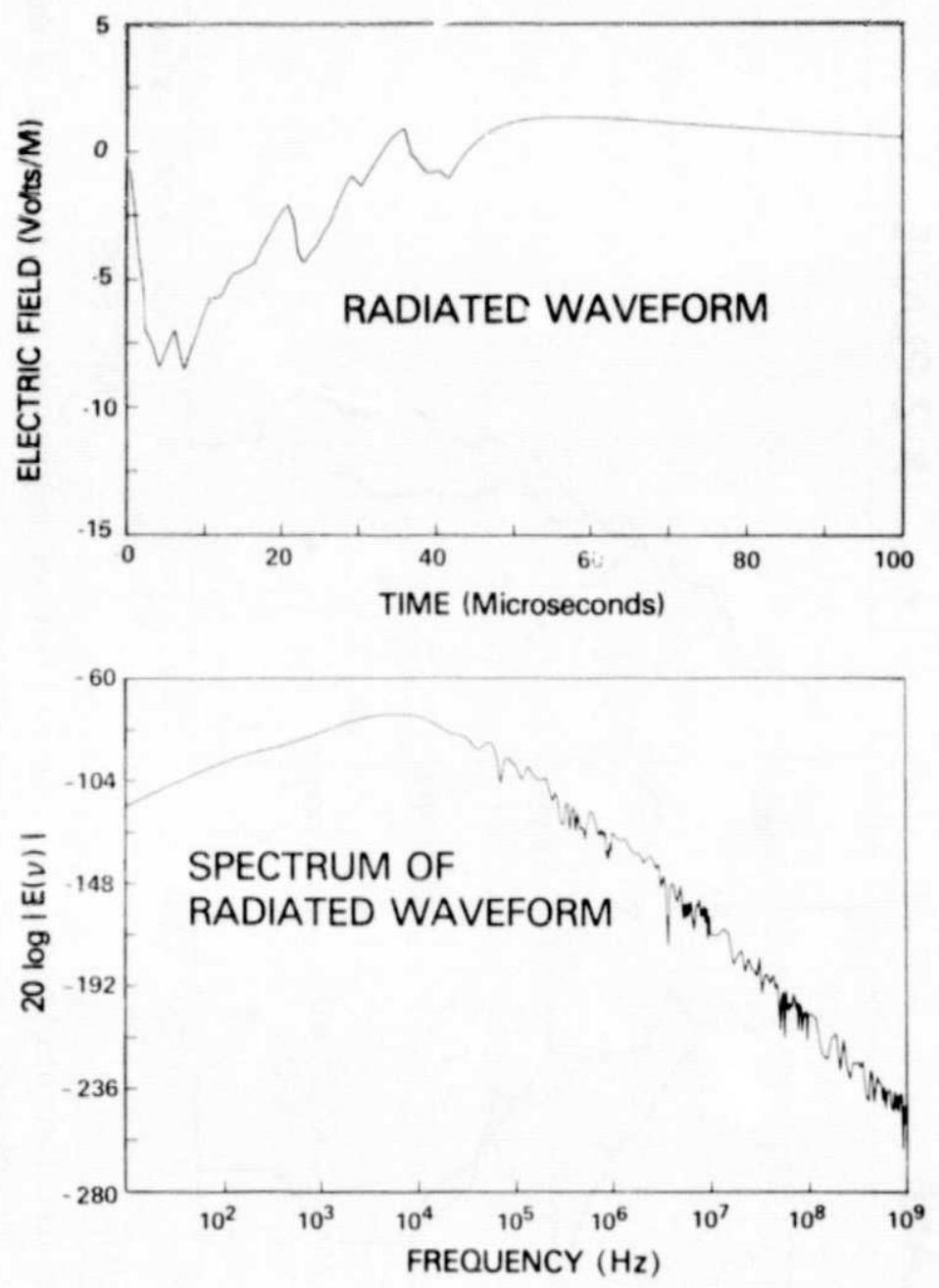

Figure 4. An example of the electric field waveform produced by the simulation in the time domain and frequency domain. The channel used is that shown in Figure 3 and the current waveform is as given in Figure 3. The velocity of propagation of the current pulse is $\mathrm{v}=\mathrm{c}$ and the observer is on the $\mathrm{y}$-axis, 100 $\mathrm{km}$ away. 


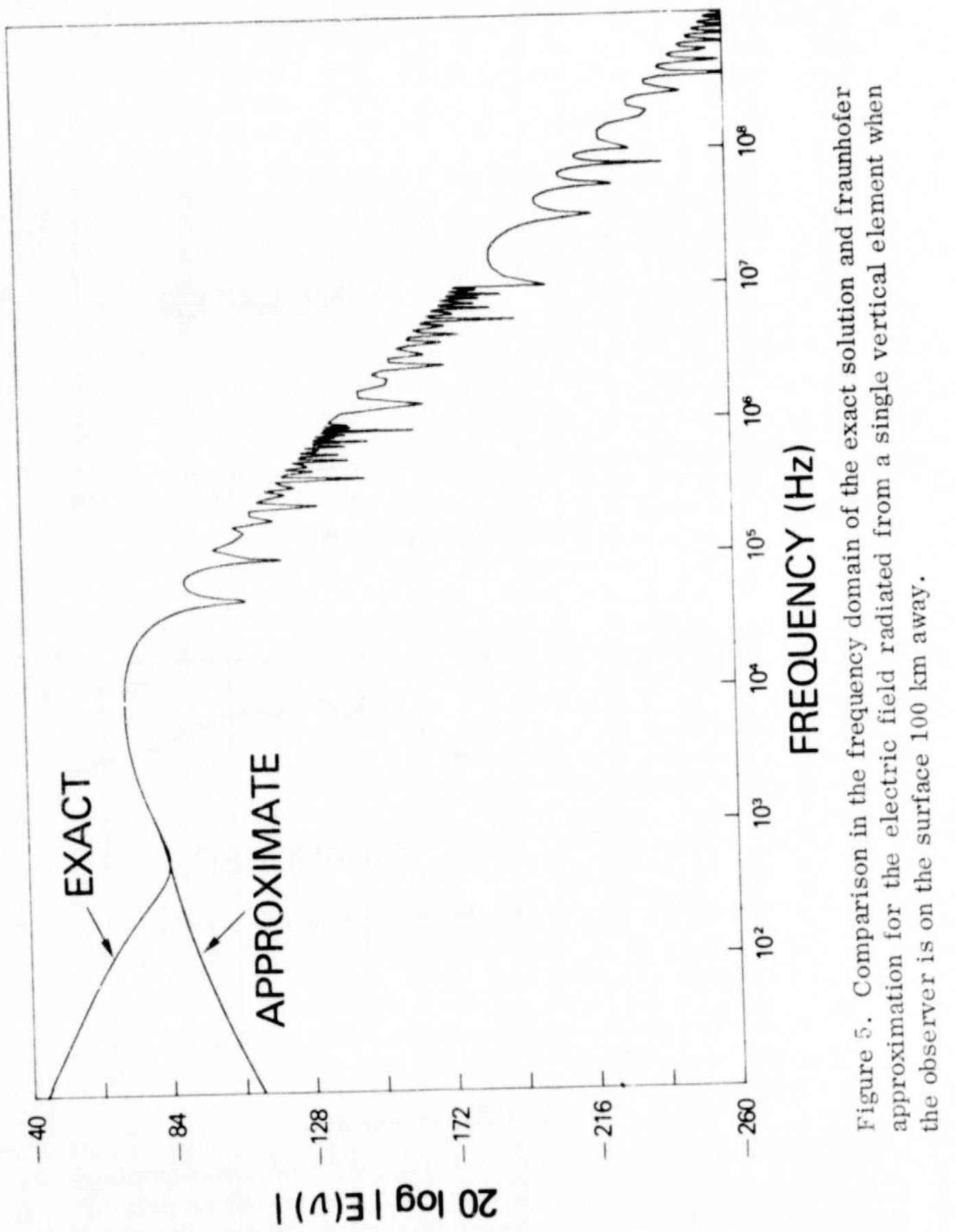



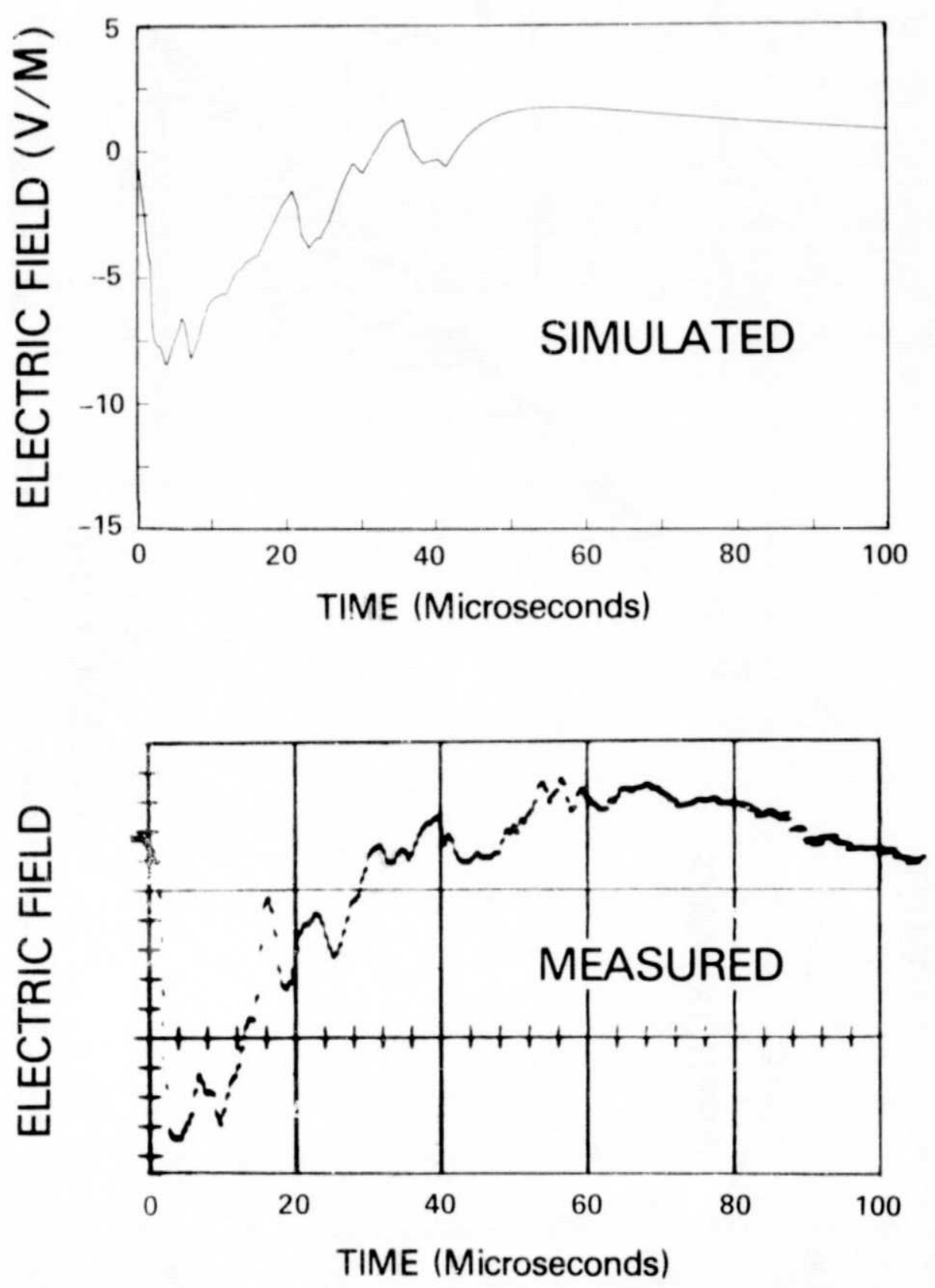

Figure 6. Comparison of simulated electric field waveform (top) with a measured waveform (bottom). The measured data represents radiation from a first return stroke at KSC, Florida. 

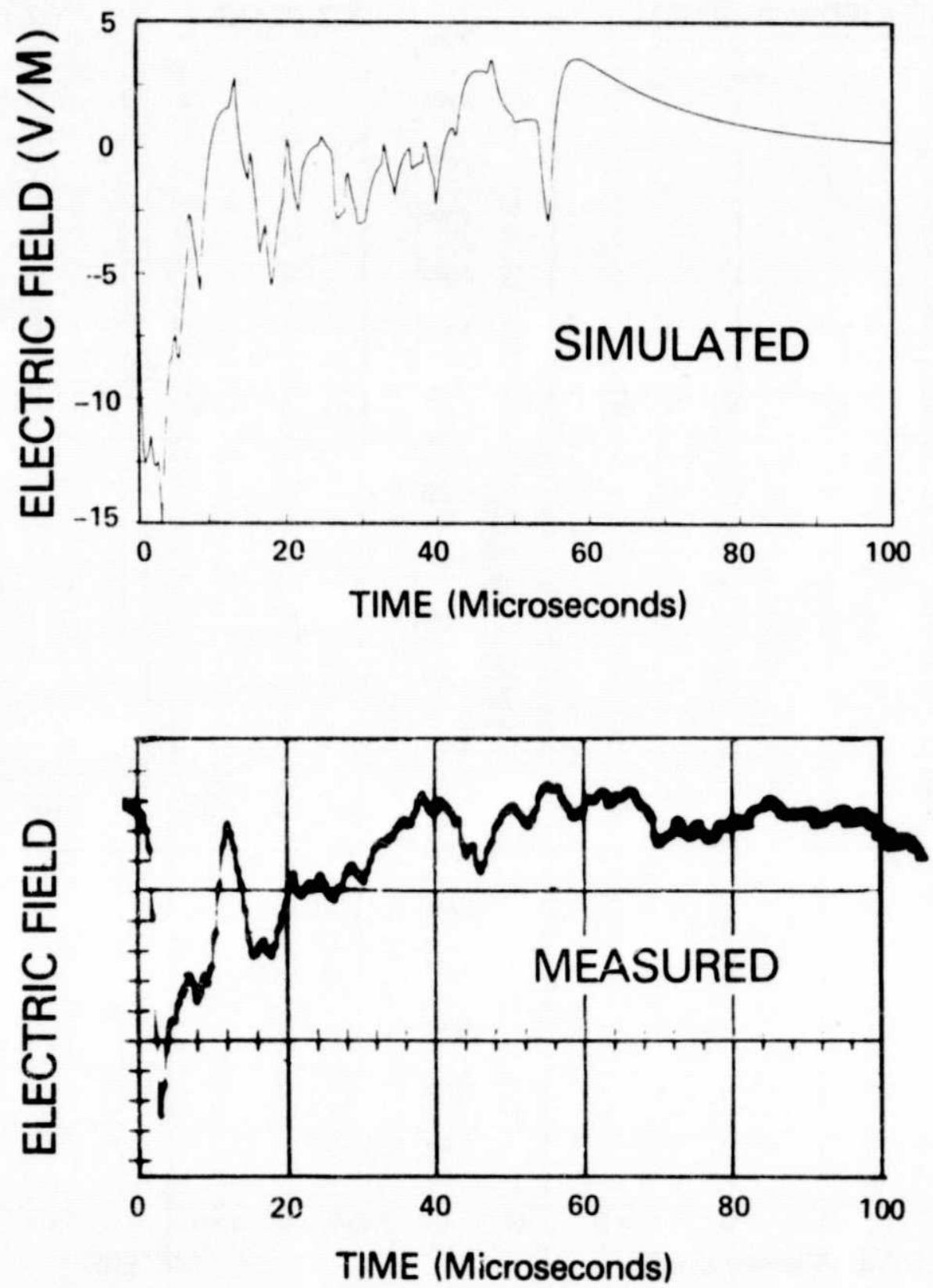

Figure 7. Comparison of simulated electric field waveform (top) with measured waveform (bottom). The measured data are from first return strokes observed at KSC, Florida. 

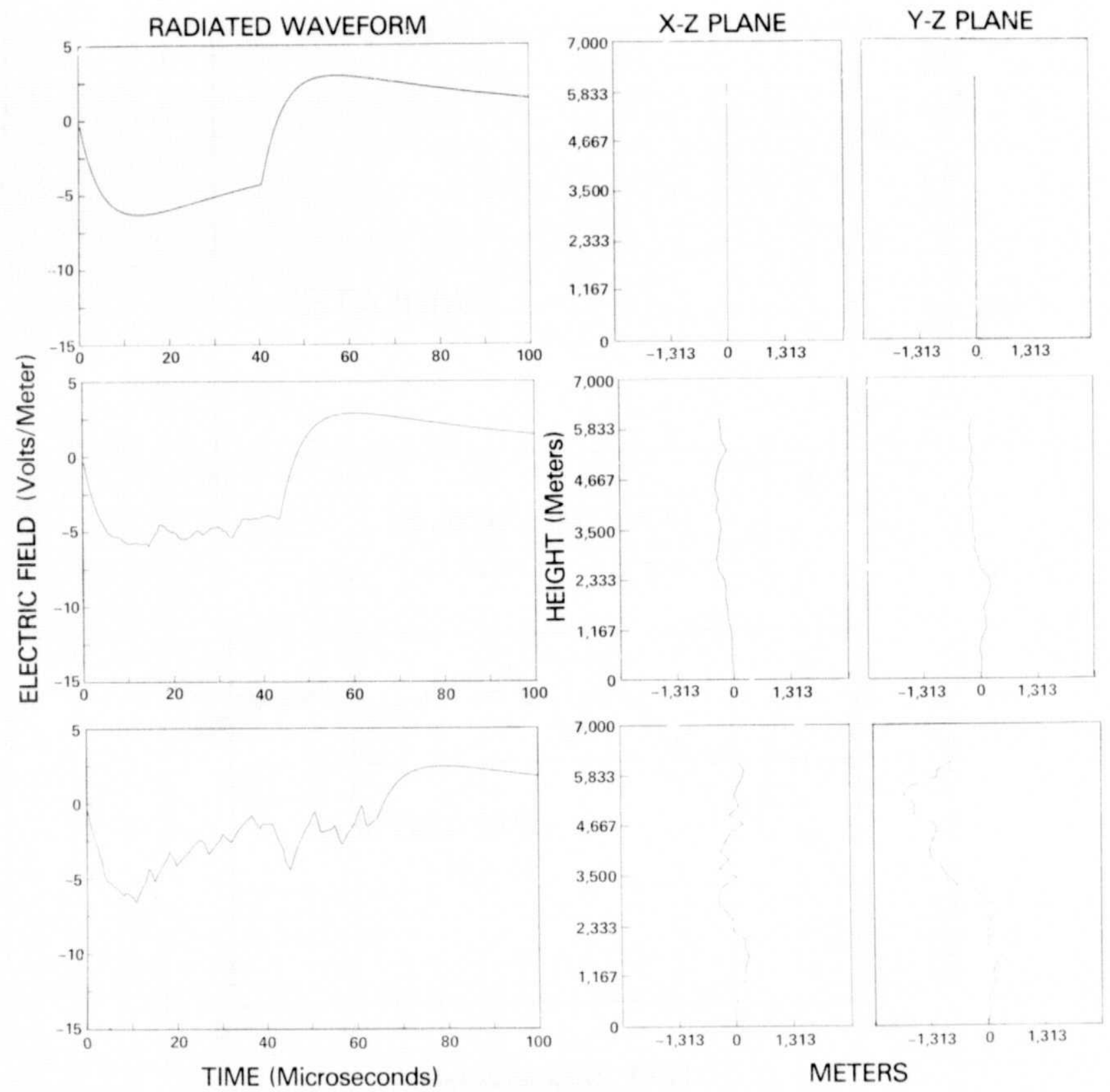

Figure 8 . The effects of increasing tortuousity on the radiated electric field waveform. 


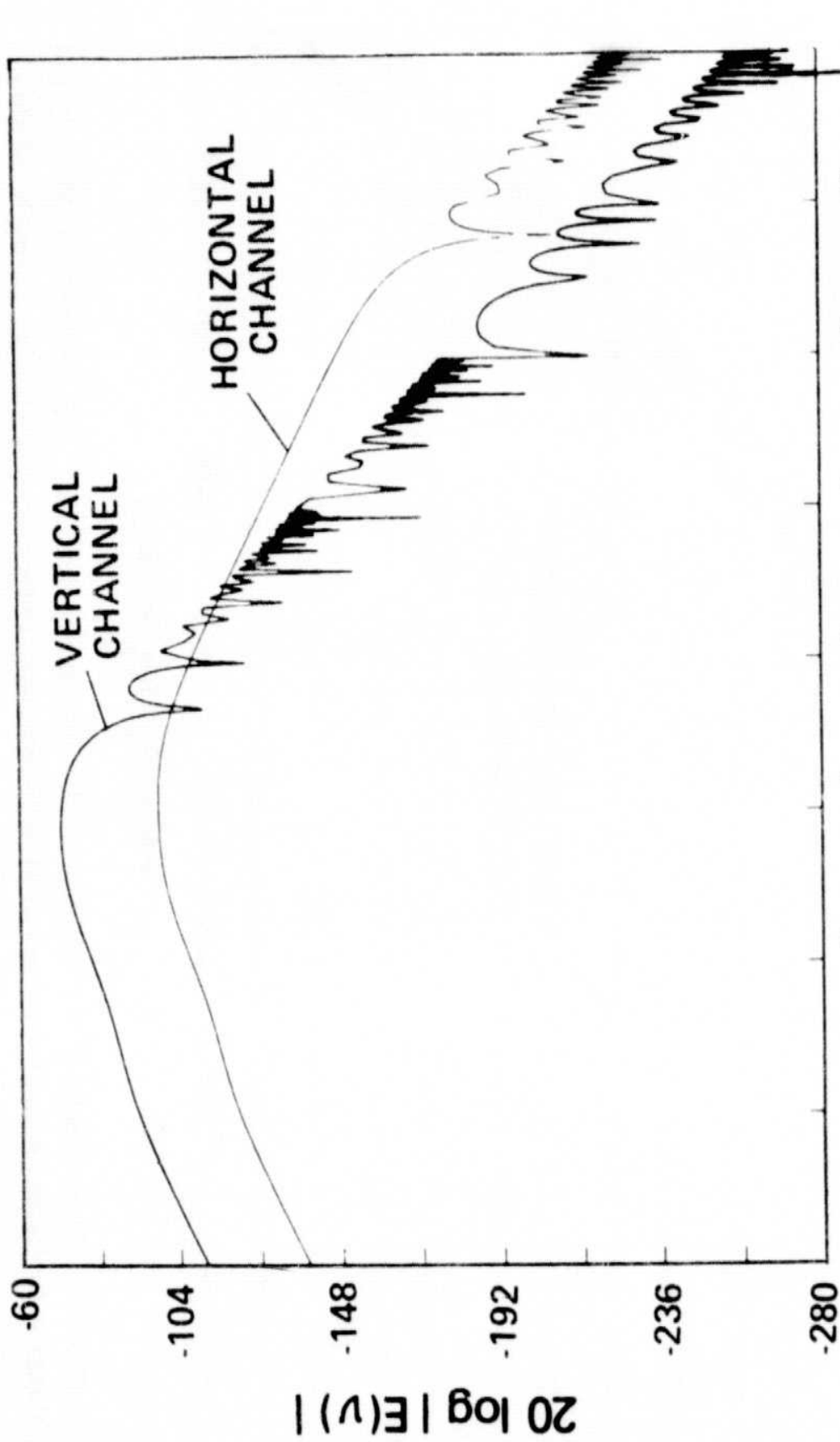

잉

ॠ

.

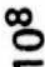

论 ญ

ช

䒿 है

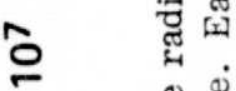

両

0

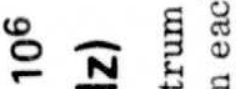

I

क

० 0 क ज

은

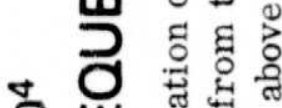

으 U

- $\boldsymbol{\sim}$ 토 호

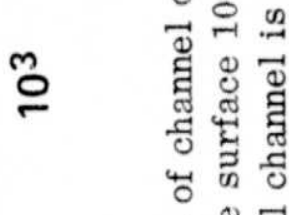

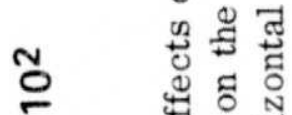

廿 ㄷำ

(1) 옹

E $\overbrace{0} \frac{1}{2}$

- 5 岳

$\sigma$ के

๑ م

苛焉

| (n) $\exists \mid$ bo 02 


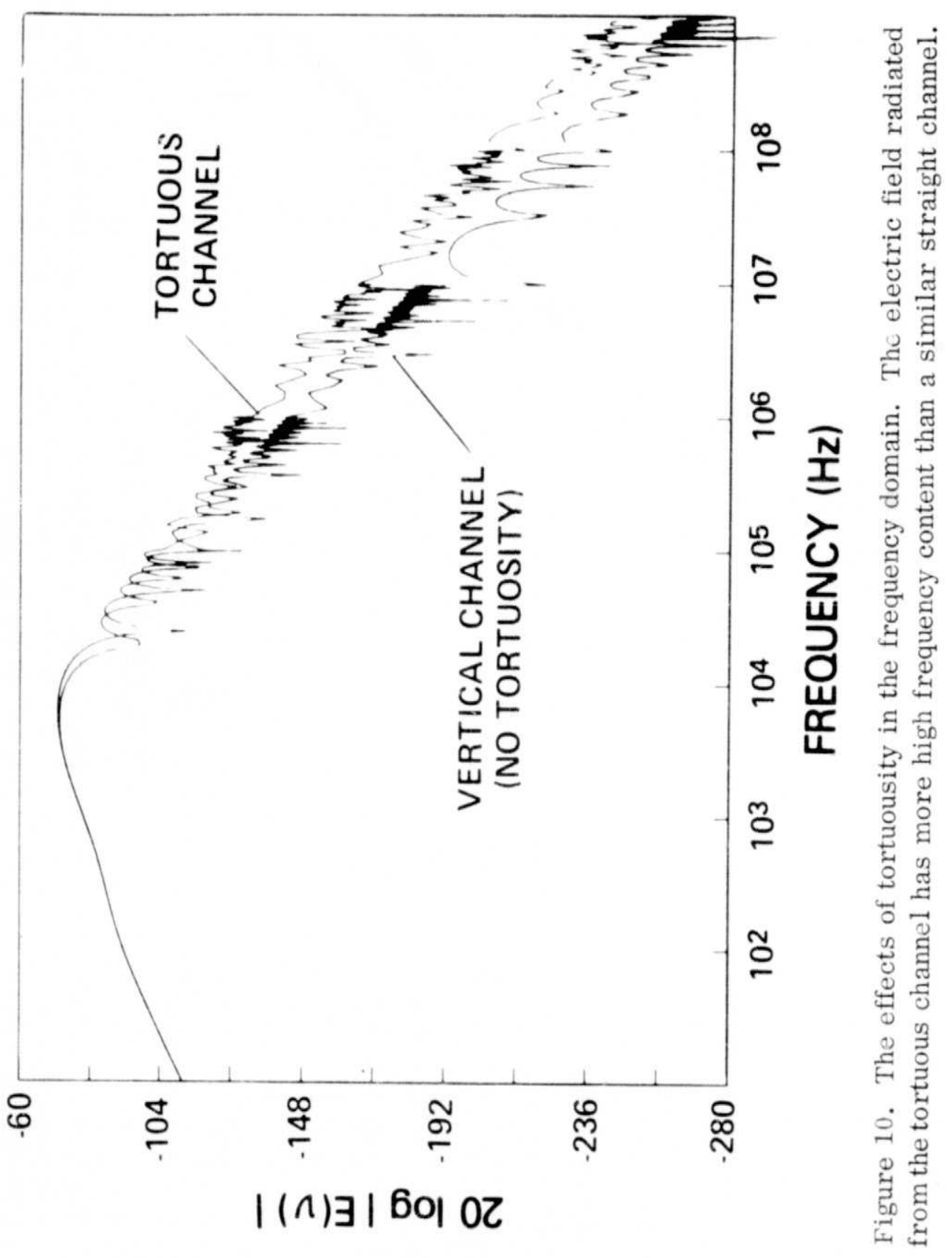

